\title{
Guiding Blind Transmitters: Relay-aided Interference Alignment for the $\mathrm{X}$ channel
}

\author{
Ye Tian and Aylin Yener \\ Wireless Communications and Networking Laboratory \\ Electrical Engineering Department \\ The Pennsylvania State University, University Park, PA 16802 \\ yetian@psu.edu_yener@ee.psu.edu
}

\begin{abstract}
This paper investigates the impact of half-duplex relays on the degrees of freedom (DoF) of the $K$-user $\mathrm{X}$ channel when the transmitters are blind in the sense that no channel state information (CSI) at the transmitter (CSIT) is available. It is shown that adding relay nodes with CSI to the communication model is sufficient to recover the optimal DoF with CSIT. In particular, the relay nodes help steer the directions of the transmitted signals to facilitate interference alignment to achieve optimal DoF. It is shown that one relay node with $K-1$ antennas is sufficient to achieve the optimal DoF. When relays do not have multiple antennas, it is sufficient to have $(K-1)^{2}$ relays to achieve the optimal DoF. The achievability is established using partial interference alignment and joint beamforming. DoF for the case when relays only have delayed CSI is also investigated.
\end{abstract}

\section{INTRODUCTION}

Interference alignment has been shown to be a useful technique to achieve the optimal degrees of freedom (DoF) for various wireless network models [1]-[3]. To effectively implement interference alignment, it is crucial to have global instant channel state information (CSI) at the transmitters (CSIT), denoted as full CSI/CSIT here onward, and is usually hard to obtain for practical systems.

When there is no CSIT in the system, loss of DoF is observed for many scenarios of interest in references [4], [5]. Recognizing the difficulty of obtaining full CSIT, references [6], [7] have studied the cases when only the channel correlation structure is known at the transmitters and have proposed interference alignment schemes using the channel correlation to achieve the optimal DoF. On the other hand, considering the time varying nature of wireless channels and the delay in the feedback of CSI, reference [8] has investigated the $K$-user broadcast channel, where CSIT is known at the transmitters with one slot delay. Under this assumption, it is shown that delayed CSIT can provide DoF gain compared to the case when no CSIT is available. However, it cannot achieve the same optimal DoF as when full CSIT is available. This delayed CSIT assumption is also applied to other channel models, and the study on its impact to the DoF can be found in [9], [10] and the references therein.

Relaying, although useful to improve the achievable rates for many wireless networks, is shown to be unable to provide DoF gain for fully connected interference channel and $\mathrm{X}$ channel with full CSI at all nodes [11]. In this context, relaying is only shown to be useful to facilitate interference alignment for some specific scenarios. For quasi-static channels, references [12]-[14] have proposed strategies to utilize the relay to randomize the channel coefficients at the receivers, and the optimal DoF can be achieved although the channel is not time varying within the transmission blocks. Reference [15] has proposed relay-aided interference alignment schemes that can achieve the optimal DoF of the $K$-user interference channel with finite time extensions. For networks that are not fully connected, the study on DoF for multi-hop relay networks can be found in reference [16]. For networks that have limited CSI, it is shown in reference [17] that using a relay, the maximum DoF for the $K$-user interference channel can be achieved when all the nodes only have local CSI, provided that the relay has more antennas than the total number of single-antenna transmitters.

Given all these efforts, it is interesting to ask whether relaying can help to achieve the maximum DoF, when no CSIT is available in the channel. Our recent work [18] has considered the 2-user X channel without CSIT, and has shown that a half-duplex relay can facilitate interference alignment to achieve the optimal DoF $\frac{4}{3}$. This can be accomplished using either (i) a relay with two antennas and delayed CSI, or (ii) a relay with one antenna and full CSI. In this work, we consider the general $K$-user $\mathrm{X}$ channel with half-duplex relays, and we show that when relays have full CSI, the optimal DoF $\frac{K^{2}}{2 K-1}$ can be achieved using one relay with $K-1$ antennas, or $(K-1)^{2}$ single-antenna relays. For the case when the relay has $K-1$ antennas, we develop a transmission scheme using partial interference alignment at the relay and joint beamforming between the relay and the transmitters to achieve the optimal DoF. For the case when relays only have one antenna, we use joint beamforming between the transmitters and the relays to align the interference at the receivers. We further show that when the relays only have delayed CSI, the maximum DoF is strictly less than $\frac{K^{2}}{2 K-1}$ in general except for the case when $K=2$.

\section{SySTEM MODEL}

For the $K$-user $\mathrm{X}$ channel, we have $K$ transmitter-receiver pairs, and each transmitter has a message to be communicated with each receiver. It is assumed that the transmitters and 
receivers are equipped with a single antenna. There are $J$ half-duplex relays with $L$ antennas available to help the transmission. We denote $w_{n m}$ as the message from transmitter $m=1, \cdots, K$ to receiver $n=1, \cdots, K$. The transmitted signal from transmitter $m$ is denoted as $X_{m}(t) \in \mathbb{C}$ and the transmitted signal from relay $R_{j}$ is denoted as $\mathbf{X}_{R_{j}}(t) \in \mathbb{C}^{L}$, where $j=1, \cdots, J$ and $t$ is the time index denoting the slot in which the signal is transmitted.

When the relays listen to the channel, the received signals at the receivers are

$$
Y_{n}(t)=\sum_{m=1}^{K} h_{n m}(t) X_{m}(t)+Z_{n}(t)
$$

and the received signals at the relays are

$$
Y_{R_{j}}(t)=\sum_{m=1}^{K} \mathbf{h}_{R_{j} m}(t) X_{m}(t)+\mathbf{Z}_{R_{j}}(t) .
$$

When the relays transmit, the received signals at the receivers are

$$
Y_{n}(t)=\sum_{m=1}^{K} h_{n m}(t) X_{m}(t)+\sum_{j=1}^{J} \mathbf{h}_{n R_{j}}(t)^{T} \mathbf{X}_{R_{j}}(t)+Z_{n}(t) .
$$

In the above expressions, the transmitted signals are subject to average power constraint $E\left(\left\|\mathbf{X}_{R_{j}}(t)\right\|^{2}\right) \leq P$, $E\left(\left|X_{m}(t)\right|^{2}\right) \leq P . h_{n m} \in \mathbb{C}$ is the channel coefficient from transmitter $m$ to the receiver $n . \mathbf{h}_{R_{j} m}(t) \in \mathbb{C}^{L}$ is the channel vector between transmitter $m$ and relay $R_{j}$, and $\mathbf{h}_{n R_{j}}(t) \in \mathbb{C}^{L}$ is the channel vector between relay $R_{j}$ and receiver $n$. It is assumed that the channel coefficients are independently drawn from a continuous distribution for each time index, and the channel is time varying. $Z_{n}(t)$ and $\mathbf{Z}_{R_{j}}(t)$ are Gaussian random variable with zero mean and unit variance, and Gaussian random vector with zero mean and identity covariance matrix, respectively.

We assume the rate of message $w_{i j}$ is $R_{i j}(P)$ for power constraint $P$. We define $\mathcal{C}(P)$ as the set of all achievable rate tuples $\left\{R_{n m}(P)\right\}$ for power constraint $P$. The degree of freedom is defined as

$$
D o F=\lim _{P \rightarrow \infty} \frac{R_{\sum}(P)}{\log (P)},
$$

where $R_{\sum}(P)=\max _{\mathcal{C}(P)}\left(\sum_{m, n} R_{n m}(P)\right)$.

\section{III. $K$-User X Channel With one Multi-Antenna RELAY}

For the $K$-user $\mathrm{X}$ channel without CSIT, it is shown in reference [5] that there is loss of DoF compared to the case with full CSIT. We show that, with one relay having $K-1$ antennas, we are able to align the interference at the receivers to achieve the DoF $\frac{K^{2}}{2 K-1}$, which is the maximum DoF when CSIT is available in the system [3], [11].

To better illustrate the transmission strategy, we first provide an example for the 3 -user $X$ channel with a relay having 2 antennas, and then generalize the result to the $K$-user case.
Proposition 1: For the 3-user $\mathrm{X}$ channel, when there is no CSIT, maximum DoF $\frac{9}{5}$ is achievable using a relay with 2 antennas and full CSI.

Proof: We assume that the data stream from transmitter $i$ to receiver $j$ is denoted as $d_{j i}, i, j=1,2,3$. To achieve the DoF $\frac{9}{5}$, we use a transmission scheme that spans 5 slots, in which a total of 9 independent data streams can be transmitted to the receivers, and each receiver can distinguish 3 of the data streams in an interference-free fashion. We also assume that the channel is time varying, i.e., each slot has a different channel realization, and the channel coefficients are drawn from a continuous distribution.

In the first 3 slots, the transmitters send messages to the receivers, and the relay keeps silent. Specifically, in slot $t$, all the 3 transmitters send the messages intended for receiver $t$ : $X_{k}(t)=d_{t k}$, where $t, k=1,2,3$. The received signals at the receivers and the relay for slot $t$ are

$$
\begin{aligned}
& Y_{m}(t)=h_{m 1}(t) d_{t 1}+h_{m 2}(t) d_{t 2}+h_{m 3}(t) d_{t 3}, \\
& \mathbf{Y}_{R}(t)=\mathbf{h}_{R 1}(t) d_{t 1}+\mathbf{h}_{R 2}(d)_{t 2}+\mathbf{h}_{R 3}(t) d_{t 3}
\end{aligned}
$$

where $m=1,2,3$, and the noise is omitted since we are considering the DoF.

In the remaining 2 slots, we need to provide each receiver with another two equations such that the intended messages, which are unknown variables $d_{t k}$, can be recovered. In the meantime, all the interference data streams must be kept in a 2-dimensional space at each receiver to achieve the maximum DoF. Since the relay only has 2 antennas, it cannot separate the 3 messages from the transmitters at each slot. However, as we show next, the spatial dimensions available at the relay can still be used to align the interference.

The relay first performs linear transformation to the received signals. For the signal received in slot 1 , we choose vectors $\mathbf{u}_{2}(1), \mathbf{u}_{3}(1) \in \mathbb{C}^{2}$ such that

$$
\begin{array}{ll}
\mathbf{u}_{2}(1)^{T} \mathbf{h}_{R 2}(1)=h_{22}(1) & \mathbf{u}_{2}(1)^{T} \mathbf{h}_{R 3}(1)=h_{23}(1) \\
\mathbf{u}_{3}(1)^{T} \mathbf{h}_{R 2}(1)=h_{32}(1) & \mathbf{u}_{3}(1)^{T} \mathbf{h}_{R 3}(1)=h_{33}(1) .
\end{array}
$$

Since we have two variables with two equations and the channel coefficients are drawn from a continuous distribution, we can guarantee the existence of $\mathbf{u}_{2}(1)$ and $\mathbf{u}_{3}(1)$ almost surely. We can then obtain the following signals by taking the inner products between the vector $\mathbf{u}_{2}(1)\left(\mathbf{u}_{3}(1)\right)$ and the received signal vector from slot 1 at the relay:

$\mathbf{u}_{2}(1)^{T} \mathbf{Y}_{R}(1)=\mathbf{u}_{2}(1)^{T} \mathbf{h}_{R 1}(1) d_{11}+h_{22}(1) d_{12}+h_{23}(1) d_{13}$ $\mathbf{u}_{3}(1)^{T} \mathbf{Y}_{R}(1)=\mathbf{u}_{3}(1)^{T} \mathbf{h}_{R 1}(1) d_{11}+h_{32}(1) d_{12}+h_{33}(1) d_{13}$.

These two signals are useful for receiver 1, since they contain the messages that are intended for it. However, the messages $d_{1 i}, i=1,2,3$ are interference for receiver 2 and 3 . Using the linear transformation provided by vector $\mathbf{u}_{2}(1)$ and $\mathbf{u}_{3}(1)$, part of the interference is aligned at receiver 2 and receiver 3 . Note that we need to keep $\mathbf{u}_{2}(1)^{T} \mathbf{Y}_{R}(1)$ away from receiver 3 , and keep $\mathbf{u}_{3}(1)^{T} \mathbf{Y}_{R}(1)$ away from receiver 2 , in order to not introducing additional interference 
to the receivers. This can be done by sending $\mathbf{u}_{2}(1)^{T} \mathbf{Y}_{R}(1)$ and $\mathbf{u}_{3}(1)^{T} \mathbf{Y}_{R}(1)$ in the direction orthogonal to $\mathbf{h}_{3 R}\left(t^{\prime}\right)$ and $\mathbf{h}_{2 R}\left(t^{\prime}\right)$, respectively, where $t^{\prime}=4,5$. We can obtain the vectors $\mathbf{u}_{1}(2), \mathbf{u}_{3}(2), \mathbf{u}_{1}(3), \mathbf{u}_{2}(3)$ in the same fashion, which result in the following inner products:

$$
X_{R}^{i}(t)=\mathbf{u}_{i}(t)^{T} \mathbf{Y}_{R}(t)=\mathbf{u}_{i}(t)^{T} \mathbf{h}_{R t}(t) d_{t t}+\sum_{k \neq t} h_{i k}(t) .
$$

In order to transmit the signals along their intended directions, we now define the following beamforming vectors $\mathbf{v}_{i j}\left(t^{\prime}\right)$ such that:

$$
\mathbf{v}_{i j}\left(t^{\prime}\right) \perp \mathbf{h}_{k R}\left(t^{\prime}\right),
$$

where $i, j, k=1,2,3$ and $i, j \neq k$. If we choose the vectors such that they have unit power, then we have $\mathbf{v}_{31}\left(t^{\prime}\right)=$ $\mathbf{v}_{13}\left(t^{\prime}\right)=\mathbf{v}_{2}^{\perp}\left(t^{\prime}\right), \mathbf{v}_{12}\left(t^{\prime}\right)=\mathbf{v}_{21}\left(t^{\prime}\right)=\mathbf{v}_{3}^{\perp}\left(t^{\prime}\right), \mathbf{v}_{23}\left(t^{\prime}\right)=$ $\mathbf{v}_{32}\left(t^{\prime}\right)=\mathbf{v}_{1}^{\perp}\left(t^{\prime}\right)$.

Using the linear transformation and beamforming vectors provided above, interference is only partially aligned. To align the rest of the interference, we let the relay choose a scaling factor $\alpha_{i j}(t)$ for each signal it wishes to send to the receivers, which results in the following signal $\mathbf{X}_{R}(t)$ to be transmitted for slot $t^{\prime}$ :

$$
\mathbf{X}_{R}\left(t^{\prime}\right)=\sum_{t=1}^{3} \sum_{i \neq t} \alpha_{t i}\left(t^{\prime}\right) \mathbf{v}_{j}^{\perp}\left(t^{\prime}\right) \mathbf{u}_{i}(t)^{T} \mathbf{Y}_{R}(t) .
$$

where $j \neq t, j \neq i$, and $\alpha_{t i}\left(t^{\prime}\right)$ are to be determined later.

For these two slots, the transmitters also send the following signals to the receivers:

$$
X_{k}\left(t^{\prime}\right)=d_{k k}
$$

where $k=1,2,3$.

The received signals at the receivers are

$$
\begin{aligned}
& Y_{m}\left(t^{\prime}\right)= \\
& h_{m 1}\left(t^{\prime}\right) d_{11}+h_{m 2}\left(t^{\prime}\right) d_{22}+h_{m 3}\left(t^{\prime}\right) d_{33}+\mathbf{h}_{m R}\left(t^{\prime}\right)^{T} \mathbf{X}_{R}\left(t^{\prime}\right)
\end{aligned}
$$

We now focus on all the signals received at receiver 1 . If we combine all the received signals from 5 slots into a vector $\mathbf{Y}_{1} \in \mathbb{C}^{5}$, we have

$$
\mathbf{Y}_{1}=\sum_{i=1}^{3}\left(\sum_{j=1}^{3} \mathbf{g}_{i j} d_{i j}\right)
$$

Due to limited space, we do not list all the vectors $\mathbf{g}_{i j}$ here. The details can be found in [19]. We only inspect the vectors $\mathbf{g}_{2 j}, j=1,2,3$ for interference data streams $d_{2 j}$ :

$$
\mathbf{g}_{21}=\left(\begin{array}{c}
0 \\
h_{11}(2) \\
0 \\
\alpha_{21}(4) h_{1 R}^{\perp 3}(4) h_{11}(2) \\
\alpha_{21}(5) h_{1 R}^{\perp 3}(5) h_{11}(2)
\end{array}\right)
$$

$$
\begin{gathered}
\mathbf{g}_{22}=\left(\begin{array}{c}
0 \\
h_{12}(2) \\
0 \\
h_{12}(4)+\alpha_{21}(4) h_{1 R}^{\perp 3}(4) \mu_{1}^{R 2}(2) \\
h_{12}(5)+\alpha_{21}(5) h_{1 R}^{\perp 3}(5) \mu_{1}^{R 2}(2)
\end{array}\right) \\
0 \\
\mathbf{g}_{23}=\left(\begin{array}{c}
h_{13}(2) \\
0 \\
\alpha_{21}(4) h_{1 R}^{\perp 3}(4) h_{13}(2) \\
\alpha_{21}(5) h_{1 R}^{\perp 3}(5) h_{13}(2)
\end{array}\right)
\end{gathered}
$$

where we denote $h_{k R}^{\perp i}(t)=\mathbf{h}_{k R}(t)^{T} \mathbf{v}_{i}^{\perp}(t), \mu_{k}^{R i}(t)=$ $\mathbf{u}_{k}(t)^{T} \mathbf{h}_{R i}(t)$.

From the above expressions, we can see that the data streams $d_{21}$ and $d_{23}$ are already aligned together. To align the data stream $d_{22}$ with $d_{21}$ and $d_{23}$, we choose

$$
\alpha_{21}\left(t^{\prime}\right)=\frac{h_{12}\left(t^{\prime}\right)}{\left(h_{12}(2)-\mu_{1}^{R 2}(2)\right) h_{1 R}^{\perp 3}\left(t^{\prime}\right)} .
$$

The signals $d_{31}, d_{32}$, and $d_{33}$ can be aligned in a similar fashion. The rest parameters can be determined using the same method for the other receivers. It is easy to verify that the data streams $d_{11}, d_{12}$ and $d_{13}$ occupy the rest 3 dimensional space. Similar argument holds for receiver 2 and receiver 3 as well. Therefore using the proposed scheme, we can transmit a total of 9 messages using 5 slots, which proves the achievability of DoF $\frac{9}{5}$.

In the transmission scheme, we can see from equation (14) that if the transmitters remain silent for slots 4 and 5, the parameters $\alpha_{i j}(t)$ will be all zero to keep the interference aligned. This reduces the total number of dimensions of the signal space at the receivers to 3 . The reason is that, using the transmission scheme provided above, each set of data streams intended for one receiver is already aligned in the first 3 slots. To keep all the interference signals aligned in a space of dimension 2, the relay may choose to do not send anything, if the transmitters remain silent for the last 2 slots. This will also keep the intended data streams in a one dimensional space. However, we need additional signal dimensions to decode the intended data streams.

To address this issue, we let the transmitters also send data streams to the receivers for slots 4 and 5 . With the additional data streams, the relay cannot keep the interference aligned by remaining silent. We call the transmission scheme joint beamforming.

The essence of the above transmission strategy is to use the limited spatial dimensions available at the relay to first partially align the interference, and then align the rest interference through joint beamforming with the transmitters.

Using the ideas from the example for the 3-user X channel with a 2-antenna relay, we can now generalize the result to the $K$-user case.

Theorem 1: For K-user X channel, when there is no CSI available at the transmitters while full CSI is available at the receivers, maximum $\operatorname{DoF} \frac{K^{2}}{2 K-1}$ is achievable using a relay with $K-1$ antennas and full CSI. 
Proof: The proof follows from similar idea as in Proposition 1 and is thus omitted here. Details can be found in [19].

Remark 1: For the $K$-user $\mathrm{X}$ channel without CSIT and relays, reference [5] has shown that the DoF is upperbounded by 1 under Rayleigh fading. Our result shows that relaying is useful to increase the DoF of $\mathrm{X}$ channel without the presence of CSIT.

Remark 2: Reference [3] has shown that with full CSI at all nodes, the maximum DoF of the $K$-user $\mathrm{X}$ channel is $\frac{K^{2}}{2 K-1}$. Reference [11] has further shown that relaying does not increase the DoF of $\mathrm{X}$ channels, when all nodes are equipped with full CSI. This means that the $K$-user $\mathrm{X}$ channel with relays has maximum DoF $\frac{K^{2}}{2 K-1}$. Our scheme has shown that without CSIT, we are still able to achieve the maximum DoF with the help of a relay.

\section{IV. $K$-user X Channel with $J$ Single Antenna RELAYS}

In the previous section, we have shown that for the $K$-user $\mathrm{X}$ channel without CSIT, it is sufficient to have one relay with $K-1$ antennas to achieve the maximum DoF of the channel. In this section, we investigate the case when relays only have a single antenna, and we study how many relays are needed to achieve the maximum DoF.

Theorem 2: For the $K$-user $\mathrm{X}$ channel with single antenna relays, when there is no CSIT but full CSI is available at the relays and the receivers, a sufficient condition to achieve the maximum DoF $\frac{K^{2}}{2 K-1}$ is to have $J=(K-1)^{2}$ relays.

Proof: For slots $t=1,2, \cdots, K$, the transmitters send the messages intended for receiver $t$, and the relays remain silent. The signal sent from transmitter $k=1,2, \cdots, K$ for slot $t$ can be expressed as

$$
X_{k}(t)=d_{t k} .
$$

The received signals at receiver $m=1,2, \cdots, K$ and relay $R_{i}, i=1,2, \cdots, J$ are

$$
\begin{gathered}
Y_{m}(t)=\sum_{k=1}^{K} h_{m k}(t) d_{t k}, \\
Y_{R_{i}}(t)=\sum_{k=1}^{K} h_{R_{i} k}(t) d_{t k} .
\end{gathered}
$$

We can see that the data streams $d_{t k}, k=1, \cdots, K$ are aligned in a one dimensional space for each $t$. For slots $t^{\prime}=$ $K+1, \cdots, 2 K-1$, we need to provide the destinations with another $K-1$ dimensions in the signal space to decode the desired $K$ messages and in the meantime keep the interference signals aligned. Using the idea of joint beamforming, each relay transmits a scaled version of the signals it received from previous time slots, i.e., for slot $t^{\prime}$, relay $R_{i}$ transmits

$$
X_{R_{i}}\left(t^{\prime}\right)=\sum_{t=1}^{K} \alpha_{i t}\left(t^{\prime}\right) Y_{R_{i}}(t),
$$

and transmitter $k$ also transmits

$$
X_{k}\left(t^{\prime}\right)=d_{k k} \text {. }
$$

The received signals at receiver $m$ for slot $t^{\prime}$ is thus

$$
Y_{m}\left(t^{\prime}\right)=\sum_{k=1}^{K} h_{m k}\left(t^{\prime}\right) d_{k k}+\sum_{i=1}^{J} h_{m R_{i}}\left(t^{\prime}\right) X_{R_{i}}\left(t^{\prime}\right) .
$$

The equation (20) can be written as

$$
\begin{aligned}
& Y_{m}\left(t^{\prime}\right)=\sum_{k=1}^{K} h_{m k}\left(t^{\prime}\right) d_{k k} \\
& +\sum_{i=1}^{J} \sum_{t=1}^{K} \sum_{k=1}^{K} h_{m R_{i}}\left(t^{\prime}\right) \alpha_{i t}\left(t^{\prime}\right) h_{R_{i} k}(t) d_{t k} .
\end{aligned}
$$

After combining the received signals at receiver $m$ from all the time slots into one vector, we have a signal in the form

$$
\begin{aligned}
& \mathbf{Y}_{m}=\mathbf{g}_{m m} d_{m m}+\sum_{k=1, k \neq m}^{K} \mathbf{g}_{m k} d_{m k} \\
& +\sum_{\gamma=1, \gamma \neq m}^{K}\left(\mathbf{g}_{\gamma \gamma} d_{\gamma \gamma}+\sum_{k=1, k \neq \gamma}^{K} \mathbf{g}_{\gamma k} d_{\gamma k}\right),
\end{aligned}
$$

where

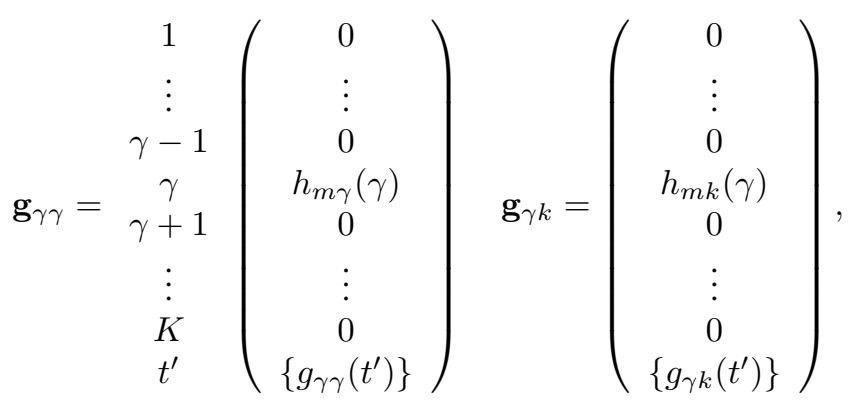

and

$$
\begin{gathered}
g_{\gamma \gamma}\left(t^{\prime}\right)=h_{m \gamma}\left(t^{\prime}\right)+\sum_{i=1}^{J} h_{m R_{i}}\left(t^{\prime}\right) \alpha_{i \gamma}\left(t^{\prime}\right) h_{R_{i} \gamma}(\gamma), \\
g_{\gamma k}\left(t^{\prime}\right)=\sum_{i=1}^{J} h_{m R_{i}}\left(t^{\prime}\right) \alpha_{i \gamma}\left(t^{\prime}\right) h_{R_{i} k}(\gamma),
\end{gathered}
$$

and $\mathbf{g}_{m m}$ and $\mathbf{g}_{m k}$ are defined in a similar fashion.

In order to separate the intended data streams from the interference, we need to align the data streams $d_{\gamma k}, k=$ $1,2, \cdots, K$ into a one dimensional space for each $\gamma \neq m$. For this end, we choose the scaling factors $\alpha_{i t}\left(t^{\prime}\right)$ such that

$$
\begin{aligned}
& \frac{\sum_{i=1}^{J} h_{m R_{i}}\left(t^{\prime}\right) \alpha_{i \gamma}\left(t^{\prime}\right) h_{R_{i} k}(\gamma)}{h_{m k}(\gamma)}= \\
& \frac{h_{m \gamma}\left(t^{\prime}\right)+\sum_{i=1}^{J} h_{m R_{i}}\left(t^{\prime}\right) \alpha_{i \gamma}\left(t^{\prime}\right) h_{R_{i} \gamma}(\gamma)}{h_{m \gamma}(\gamma)}, \quad \forall k \neq \gamma .
\end{aligned}
$$

Fixing $\gamma, t^{\prime}$ and $m$, we have $K-1$ equations for each $k \neq \gamma$. We also have $K-1$ possible values for $m \neq \gamma$. Therefore, we have $(K-1)^{2}$ equations in total for each combination of $t^{\prime}$ and 
$\gamma$, and we need $J=(K-1)^{2}$ variables in order to guarantee the existence of a solution to the set of linear equations. Since all the relays have full CSI, they can solve the equations to obtain the scaling factors individually. Using the scaling factors, it is now easy to verify that all the interfering signals are aligned into a $K-1$ dimensional space, and the intended data streams occupy the rest $K$ dimensional space, due to the fact that the channel is time varying and channel coefficients are drawn from a continuous distribution. We can then decode the intended messages using a zero-forcing decoder, and the DoF $\frac{K^{2}}{2 K-1}$ can be achieved.

\section{The DoF with Delayed CSI at the Relay}

In this section, we investigate the impact of relays on DoF when the relays only have delayed CSI. We consider the $K$ user $\mathrm{X}$ channel with one $(K-1)$-antenna relay, and compare the DoF of the model with full CSI and the one with delayed CSI. For the case with delayed CSI, we first consider the $K$ user $\mathrm{X}$ channel with a $K$-antenna relay, which clearly provides a DoF upperbound to the case with a $(K-1)$-antenna relay.

Theorem 3: For the $K$-user $\mathrm{X}$ channel with a $K$-antenna relay, when there is no CSIT and only delayed CSI is available at the relay, the maximum DoF is

$$
\frac{K}{1+\frac{1}{2}+\cdots+\frac{1}{K}}
$$

Proof: The achievability of this DoF can be obtained using a similar strategy as in [8]. The scheme in [8] is designed for the $K$-user broadcast channel and consists of $K$ phases, where in phase 1 , the transmitter sends the messages to the receivers. In slot $t=1, \cdots, K$ for phase 1 , the transmitter sends $\mathbf{X}(t)=\left(d_{t 1}, d_{t 2}, \cdots, d_{t K}\right)^{T}$, where $d_{t i}$ is the $i$ th message intended for receiver $t$. The transmission scheme used for this phase can be implemented for the $K$-user $\mathrm{X}$ channel. Since the relay has $K$ antennas and delayed CSI, it can decode all the messages, and then it can act as the transmitter in the broadcast channel to implement the transmission scheme for the rest of the phases to achieve the DoF specified by (26).

To upper bound the DoF of the channel, we combine all the transmitters and the relay, which yields a broadcast channel with $2 K$ antennas at the transmitter with delayed CSIT. The outerbounds in references [8] [9] can then be used to obtain equation (26).

Recall that for the $K$-user $\mathrm{X}$ channel without CSIT, when the relay has full CSI, we can achieve the full DoF $\frac{K^{2}}{2 K-1}$ with only $K-1$ antennas at the relay. For the case with delayed CSI at the relay, when the relay has $K-1$ antennas, the DoF at most equals equation (26). It is clear that for the $K$-user $\mathrm{X}$ channel without CSIT, full CSI at the relay can provide a DoF gain, compared to the case with delayed CSI at the relay.

\section{CONCLUSION}

In this paper, we have investigated relay-aided interference alignment schemes for $K$-user $\mathrm{X}$ channel, when no channel state information (CSI) at the transmitter (CSIT) is available. For the case when relays have full CSI, we have developed a transmission scheme based on partial interference alignment and joint beamforming, and have shown that using one relay with $K-1$ antennas, the optimal DoF can be achieved. We have also designed joint beamforming based relaying schemes for the case when relays only have a single antenna, and have shown that the optimal DoF can be achieved with $(K-1)^{2}$ relays. Degradation on the DoF when relays only have delayed CSI is also observed.

\section{REFERENCES}

[1] V. R. Cadambe and S. A. Jafar, "Interference alignment and the degrees of freedom for the K-user interference channel," IEEE Transactions on Information Theory, vol. 54, no. 8, pp. 3425-3441, Aug 2008.

[2] S. A. Jafar and S. Shamai, "Degrees of freedom region for the MIMO $\mathrm{X}$ channel," IEEE Transactions on Information Theory, vol. 54, no. 1, pp. 151-170, January 2008.

[3] V. R. Cadambe and S. A. Jafar, "Interference alignment and the degrees of freedom of wireless X networks," IEEE Transactions on Information Theory, vol. 55, no. 9, pp. 3893-3908, September 2009.

[4] C. Huang, S. A. Jafar, S. Shamai, and S. Vshwanath, "On degrees of freedom region of MIMO networks without CSIT," IEEE Transactions on Information Theory, accepted.

[5] C. S. Vaze and M. Varanasi, "The degrees of freedom regions of MIMO broadcast, interference, and cognitive radio channels with no CSIT," submitted to IEEE Transactions on Information Theory, October 2009, available: http://arxiv.org/abs/0909.5424.

[6] S. A. Jafar, "Exploiting channel correlations - simple interference alignment schemes with no CSIT," available at arXiv:0910.0555.

[7] C. Wang, T. Gou, and S. A. Jafar, "Aiming perfectly in the dark blind interference alignment through staggered antenna switching," IEEE Transactions on Signal Processing, vol. 59, no. 6, pp. 2734-2744, June 2011.

[8] M. A. Maddah-Ali and D. Tse, "Completely stale transmitter channel state information is still very useful," in Proceedings of Forty-eighth Annual Allerton Conference On Communication, Control, and Computing, September 2010.

[9] C. S. Vaze and M. K. Varanasi, "The degrees of freedom region of the two-user MIMO broadcast channel with delayed CSIT," in Proceedings of IEEE International Symposium on Information Theory, July 2011.

[10] A. Ghasemi, A. S. Motahari, and A. K. Khandani, "On the degrees of freedom of X channel with delayed CSIT," in Proceedings of IEEE International Symposium on Information Theory, July 2011.

[11] V. R. Cadambe and S. A. Jafar, "Degrees of freedom of wireless networks with relays, feedback, cooperation and full duplex operation," IEEE Transactions on Information Theory, vol. 55, no. 5, pp. 23342344, May 2009.

[12] B. Nourani, S. A. Motahari, and A. K. Khandani, "Relay-aided interference alignment for the quasi-static X channel," in Proceedings of IEEE International Symposium on Information Theory, June 2009.

[13] — , "Relay-aided interference alignment for the quasi-static interference channel," in Proceedings of IEEE International Symposium on Information Theory, June 2010.

[14] D. Jin, J. No, and D. Shin, "Interference alignment aided by relays for the quasi-static X channel," in Proceedings of IEEE International Symposium on Information Theory, July 2011.

[15] H. Ning, C. Ling, and K. K. Leung, "Relay-aided interference alignment: Feasibility conditions and algorithm," in Proceedings of IEEE International Symposium on Information Theory, June 2010.

[16] T. Gou, S. A. Jafar, S.-W. Jeon, and S.-Y. Chung, "Aligned interference neutralization and the degrees of freedom of the $2 \times 2 \times 2$ interference channel," available at arXiv:1102.3833.

[17] R. Tannious and A. Nosratinia, "Relay-assisted interference network: Degrees of freedom," IEEE Transactions on Information Theory, accepted.

[18] Y. Tian and A. Yener, "Relay-aided interference alignment for the X channel with limited CSI," in Proceedings of IEEE Wireless Communications and Networking Conference, WCNC'12, Paris, France, April 2012.

[19] $~-$, "Guiding the blind transmitters: Degrees of freedom optimal interference alignment using relays," submitted to IEEE Transactions on Information Theory, Febuary 2012. 